\title{
Influencing Factors on Measuring Surface Residual Stress of Steel Bars by X-ray Diffraction Method
}

\author{
Wu Yiwen ${ }^{1,2}$, Li Sijin ${ }^{2}$, Zhu Pei ${ }^{2}$, Wang Xiaoyu ${ }^{1}$ \\ ${ }^{1}$ School of Material Science and Engineering, Shanghai University, Shanghai, China \\ ${ }^{2}$ Inspection Centre of Industrial Products \& Raw Materials, Shanghai Customs of the People's Republic of China, Shanghai, China
}

Email address:

wumetal@sina.cn (Wu Yiwen), lisijin1229@163.com (Li Sijin)

\section{To cite this article:}

Wu Yiwen, Li Sijin, Zhu Pei, Wang Xiaoyu. Influencing Factors on Measuring Surface Residual Stress of Steel Bars by X-ray Diffraction Method. American Journal of Physics and Applications. Vol. 9, No. 5, 2021, pp. 121-126. doi: 10.11648/j.ajpa.20210905.14

Received: September 7, 2021; Accepted: October 4, 2021; Published: October 15, 2021

\begin{abstract}
The surface residual stress of mechanical parts and large mechanical components has an important influence on their fatigue strength, stress corrosion resistance and service life. X-ray diffraction method is used to measure the surface residual stress of steel bars and the influence of factors such as testing methods, testing equipment status and sample status on testing results is discussed in this paper. The results show that the testing parameters such as the target material, filter, diffraction angle and the minimum step shall be selected according to the material type and crystal structure under the premise of stable operation of the equipment in testing process. After that, the data treatment is under consideration. The peak determination is a very important part of data processing. The cross-correlation method is widely used because it uses all the original test data for the calculation, and the accuracy of the peak determination is high in this test. It also shows that the sampling process and specimen protection can also affect the testing results of the residual stress. The structure state of materials will have a great impact on testing results. For example, the texture will cause excessive errors of the testing results. It is recommended to understand the material properties in detail before testing so as to ensure sufficient margin when sampling, to apply the strict protection for the test surface of the sample, and to add a swing angle appropriately when the material is with texture.
\end{abstract}

Keywords: Steel Bar, Surface Residual Stress, X-ray Diffraction, Influencing Factor

\section{Introduction}

Residual stress generally caused by local uneven plastic deformation is a kind of elastic pressure [1]. The residual stress of mechanical parts and large mechanical components has an important influence on their fatigue strength, stress corrosion resistance, dimensional stability and service life $[2$, 3]. Appropriate and reasonably distributed residual stress can enhance the fatigue strength and stress corrosion resistance of the mechanical parts and components, thereby prolonging their service life $[4,5]$.

Currently, the common testing methods for the residual stress can generally be divided into two broad categories: mechanical destructive and non-destructive methods. There are several methods for the mechanical destructive methods such as blind-hole method, strip-cutting method and layer-by-layer milling method. Non-destructive methods include X-ray diffraction method, neutron diffraction method, magnetic method, ultrasonic method, electron scattering interference method and metal magnetic memory method [6].

The X-ray diffraction method, a non-destructive method, can be used to test the surface residual stress. This method has excellent maturity and precision. The X-ray diffraction method can measure the residual stress of the specimens in the axial, tangential and radial directions. In addition, this method can be used to test the residual stress of curved surface and spherical surface of the specimens. It is also widely used in the measurement of the film stress $[7,8]$.

The surface residual stress of round bars was measured using an X-ray stress tester of X-350A model produced by Ester Stress Technology. The effect of the selection of test parameters, the state of testing equipment, the state of the specimen and other factors on the test results are discussed. This article should serve as a valuable reference for the other relevant users. 


\section{Technical Key Points for Measuring Residual Stress by X-ray Diffraction Method}

The national standard for the determination of the residual stress by X-ray diffraction in China is GB/T 7704-2017 "Non-Destructive Testing--Practice for residual stress measurement by X-ray" [9]. An accurate understanding of the existing standards is the first step to do a good job of testing, mainly reflected in the following aspects.

\subsection{Parameters Selection}

First of all, the personnel in non-destructive testing should choose targets appropriate for the types of materials, and then the $K_{\beta}$ filter is selected on the basis of the above target. The material type, diffraction crystal plane and stress constant should also be chosen according to the specimen. The high multiplicity factor avoiding or weakening the influence of the texture should be considered for the selection of a diffraction crystal. Test conditions for the residual stress of common materials by X-ray diffraction are shown in Table 1 .

Table 1. X-ray diffraction conditions for residual stress measurement of some common materials.

\begin{tabular}{|c|c|c|c|c|c|}
\hline Alloy & Crystal Structure & Target Materials & $\mathbf{K}_{\beta}$ filter & $\{$ hkl $\}$ crystal surface & Diffraction angle $2 \theta /\left(^{\circ}\right)$ \\
\hline Nickel Alloy & Cube & $\mathrm{Mn}$ & $\mathrm{Cr}$ & $\{311\}$ & $152 \sim 162$ \\
\hline Ferrite steel cast iron (Matrix) & Cube & $\mathrm{Cr}$ & $\mathrm{V}$ & $\{211\}$ & 156 \\
\hline Austenitic steel & Cube & $\mathrm{Mn}$ & $\mathrm{Cr}$ & $\{311\}$ & 152 \\
\hline Aluminium alloy & Cube & $\mathrm{Cu}$ & $\mathrm{Ni}$ & $\{422\}$ & 137 \\
\hline Cobalt alloy & Cube & $\mathrm{Mn}$ & $\mathrm{Cr}$ & $\{311\}$ & $153 \sim 159$ \\
\hline Copper Alloy & Cube & $\mathrm{Mn}$ & $\mathrm{Cr}$ & $\{311\}$ & 149 \\
\hline Molybdenum alloys & Cube & $\mathrm{Fe}$ & $\mathrm{Mn}$ & $\{310\}$ & 153 \\
\hline Zirconium alloys & Hexagonal & $\mathrm{Fe}$ & $\mathrm{Mn}$ & $\{213\}$ & 147 \\
\hline Tungsten Alloy & Cube & $\mathrm{Co}$ & $\mathrm{Fe}$ & $\{222\}$ & 156 \\
\hline \multirow{2}{*}{$\alpha$ - Aluminium oxide } & \multirow{2}{*}{ Dense row hexagonal } & $\mathrm{Cu}$ & $\mathrm{Ni}$ & $\{146\}\{4.0 .10\}$ & 136145 \\
\hline & & $\mathrm{Fe}$ & $\mathrm{Mn}$ & $\{2.1 .10\}$ & 152 \\
\hline \multirow{2}{*}{$\beta$ - Aluminium oxide } & \multirow{2}{*}{ Cube } & $\mathrm{C}$ & $\mathrm{Ni}$ & $\{844\}$ & 146 \\
\hline & & $\mathrm{V}$ & $\mathrm{Ti}$ & $\{440\}$ & 128 \\
\hline
\end{tabular}

This test uses a scanning technique to measure the surface residual stress of steel bars. Therefore, the selection of the scanning step is aimed at obtaining a relatively smooth diffraction curve. Generally, the minimum step should not be greater than $0.1^{\circ}$. The number of $\mathrm{X}$-ray photons received by the detector in specified time is counted. The higher the count of the X-ray photons is, the smaller is the random error. The center of the test surface should be accurately placed at the coincident position of the center of the test point, the center of the X-ray spot, and the rotation center of the goniometer.

\subsection{Common Methods}

Iso-inclination method and side-inclination method are common methods for determining residual stress by X-ray diffraction [4]. In iso-inclination method, the specimen is rotated about the $\omega$ axis. Both $\omega$ and $2 \theta$ are in the same plane. It can be divided into equal inclination fixed $\Psi_{0}$ method (also called the $\omega$ method), equal inclination oscillation method and iso-inclination fixed $\Psi$ method. In side-inclination method (also called the $\chi$ method), the specimen is rotated about the $\chi$ axis. $\omega$ and $2 \theta$ are in perpendicular plane. It can be divided into side-inclination fixed $\psi$ method and side-inclination fixed $\psi$ oscillation method [10-12].

Compared with the iso-inclination method, there are some advantages for the side-inclination method. (1) In the case of limited space, it is better to use the side-inclination method; (2) The absorption factor is independent from $\Psi$ angle, so it is not necessary to make the absorption correction for the diffraction intensity; (3) for some materials, if there are no peaks or poor peaks in the higher angle range $\left(140^{\circ} \sim 170^{\circ}\right)$, the diffraction peaks with lower angles can be used $[13,14]$. The fixed $\Psi$ method is superior to the fixed $\Psi_{0}$ method due to its accurate principle and good practical effect. The implementation of the fixed $\Psi$ method under the condition of side inclination produces a superior new feature. The absorption factor is constantly equal to 1 . In other words, regardless of whether the diffraction peak is diffuse or not, its backside does not tilt and the peak shape is basically symmetrical. In addition, the peak shape and intensity do not change with $\Psi$ angle in the absence of texture. In the case of the slight texture or slightly coarse crystal, the fixed $\Psi$ method can also show its advantages.

\subsection{Treatment of the Data}

Based on the testing principle of X-ray diffraction method, $\mathrm{X}$-ray with the wavelength $\lambda$ irradiates to the specimen several times with different incident angles $\Psi$. The corresponding diffraction angle $2 \theta$ is measured, and then the internal stress $\sigma_{\Phi}$ can be calculated by the slope $M$ of $2 \theta$ to $\sin ^{2} \Psi$.

In practical applications, the common methods are the $\sin ^{2} \psi$ method and the $0^{\circ} \sim 45^{\circ}$ method. The $0^{\circ} \sim 45^{\circ}$ method is a simplified method of the $\sin ^{2} \psi$ method. In the test, the linear relationship between $2 \theta$ and $\sin ^{2} \psi$ may be unsatisfactory due to the test system or the specimen itself. The specimen should be tested multiple times to avoid large random errors. Therefore, the $\sin ^{2} \psi$ method is often used.

The peak determination is a very important part of the data processing. Before the peak determination, the background processing and intensity factor correction will be performed. 
After the peak determination, the software will also make stress value calculations and error analysis.

Common methods for the peak determination include half-width method, parabola method and cross-correlation method [15]. The half-width method is to remove the background of the diffraction curve from a certain diffraction peak, and to take the $2 \theta$ corresponding to the midpoint of the line at $1 / 2$ of the net height of the diffraction peak as the position of the diffraction peak. The parabola method is to use the least square method to determine the point at the top of the net diffraction peak (the part above $80 \%$ of the peak intensity), and to use the abscissa of the top point of the parabola as the peak position to determine the peak. The cross-correlation method is to construct a cross-correlation function curve through the diffraction peak curves measured at two different incident angles, and to use the abscissa of the maximum point of the cross-correlation curve as the peak position difference $\Delta 2 \theta$ of the two diffraction peaks. The cross-correlation method uses all the original test data for the calculation, and the accuracy of the peak determination is high. In this testing, the cross-correlation method is used to determine the peak. However, the cross-correlation method requires that the two diffraction peaks undergoing cross-correlation processing are similar in shape. If the measured material is irregularly distorted due to the texture, the coarse crystal, the material composed of multiple phases or the selected diffraction peak nearby other phase peaks, the cross-correlation method is obviously inappropriate.

\section{Equipment and Parameters for Measuring the Residual Stress by X-ray Diffraction Method}

\subsection{Equipment and Its Stability}

The stability of the equipment for the residual stress has a greater impact on the testing results. Before the test, you should check whether the various parts of the instrument are in correct position, choose a collimator tube with an appropriate diameter, select the appropriate target material according to the test materials refer to Table 1 and then use a filter whose atomic number is 1 or 2 smaller than that of the target to filter $\mathrm{K}_{\beta}$, thereby avoiding or reducing the generation of the fluorescent radiation.

Check the stability of the equipment before the test. When a test is completed, the calibration of the test center spot should be re-calibrated, and then multiple tests should be performed.

If the value of the 5 times tests has a certain deviation within the allowable range of the testing result of the equipment $( \pm 25 \mathrm{MPa})$ [9] and their arithmetic mean and standard deviation are between 0-25 $\mathrm{MPa}$, it can be found that the state of the equipment is stable. If the deviation exceeds the allowable range of the test results of the equipment, it will affect the accuracy of the testing results. So, the instrument needs to be checked to reduce the system error.

After the equipment has been running for a period of time, the deviation of the test results begins to increase. At this time, the equipment should be stopped for a period of time, and then the calibration should be carried out. The test should be performed after the above calibration. This can ensure the accuracy of the repeatability to a certain extent.

\subsection{Test Case}

To detect the distribution of the longitudinal residual stress, a test on the surface residual stress of a cylindrical pearlitic steel bar is performed. The schematic diagram of the specimen is shown in Figure 1. Based on the previous requirements, the selection of test parameters is shown in Table 2.

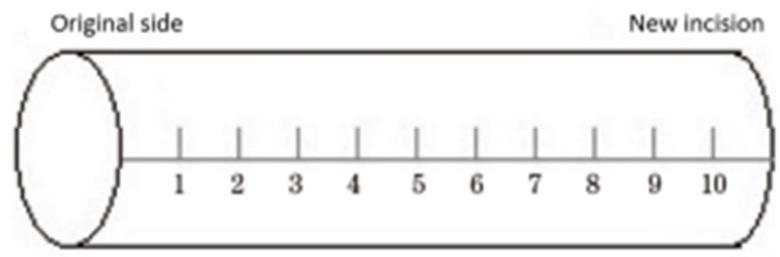

Figure 1. Schematic diagram of the specimen for residual stress.

Table 2. Testing parameters for residual stress.

\begin{tabular}{|c|c|c|c|c|c|c|c|c|c|c|c|}
\hline $\begin{array}{l}\text { Radiation } \\
\text { target }\end{array}$ & $\begin{array}{l}\text { Stress } \\
\text { calculating } \\
\text { method }\end{array}$ & $\begin{array}{l}\text { Tube } \\
\text { current } \\
\text { /mA }\end{array}$ & $\begin{array}{l}\text { diffraction } \\
\text { crystal plane }\end{array}$ & $\begin{array}{l}2 \theta \text { scan } \\
\text { start } \\
\text { angle }\end{array}$ & $\begin{array}{l}2 \theta \text { scan } \\
\text { end } \\
\text { angle }\end{array}$ & $\begin{array}{l}\text { Measuring } \\
\text { method }\end{array}$ & $\psi /\left({ }^{\circ}\right)$ & $\begin{array}{l}\text { Tube } \\
\text { voltage }\end{array}$ & $\begin{array}{l}\text { Peak searching } \\
\text { method }\end{array}$ & $\begin{array}{l}\text { Stress } \\
\text { constant } \\
/ \mathrm{MPa} \\
\end{array}$ & $\begin{array}{l}2 \theta \text { scan } \\
\text { step }\end{array}$ \\
\hline $\mathrm{CrK} \alpha$ & $\begin{array}{l}\operatorname{Sin}^{2} \psi \\
\text { method }\end{array}$ & 5.0 & $(211)$ & 162 & 151 & $\begin{array}{l}\text { side-inclination } \\
\text { fixed } \psi \text { method }\end{array}$ & $\begin{array}{l}0,24.2 \\
35.3,45.0\end{array}$ & 20 & cross-correlation & -318 & 0.10 \\
\hline
\end{tabular}

Before the testing, the equipment is zeroed using reduced iron powder. The residual stress turns out to be $-9,-7$, and -12 $\mathrm{MPa}$ respectively, which are within $\pm 20 \mathrm{MPa}$. It proves that the equipment is stable and usable.

The distribution curve of cross-correlation function tested at the point 7 of the specimen in Figure 1 is shown in Figure 2. Because there is the maximum at cross-correlation function, for any two diffraction lines, the peak difference can be learned as long as the abscissa of the maximum is calculated. The results calculated by computer are shown in Table 3 . From Table 3, it is then possible to calculate the residual stress as-311.0 $\mathrm{MPa}$ (the negative sign represents compressive stress, same below) with an error of $\pm 18 \mathrm{MPa}$.

Table 3. Calculating results of the correlation function.

\begin{tabular}{lllll}
\hline $\boldsymbol{\psi}$ & Diffraction peak position $\mathbf{2 \theta}_{\mathbf{p}}$ & Peak count & full width at half & Integral intensity \\
\hline $0^{\circ}$ & $155.984^{\circ}$ & 354 & $2.40^{\circ}$ & 1852.4 \\
$24.2^{\circ}$ & $156.166^{\circ}$ & 352 & $2.45^{\circ}$ & 1917.4 \\
\hline
\end{tabular}




\begin{tabular}{llllll}
\hline $\boldsymbol{\psi}$ & Diffraction peak position $\mathbf{2 \theta}_{\mathbf{p}}$ & Peak count & full width at half & Integral intensity & Integral breadth \\
\hline $35.3^{\circ}$ & $156.350^{\circ}$ & 350 & $2.50^{\circ}$ & 1959.8 & $2.80^{\circ}$ \\
$45.0^{\circ}$ & $156.465^{\circ}$ & 348 & $2.39^{\circ}$ & 1832.6 & $2.63^{\circ}$ \\
\hline
\end{tabular}

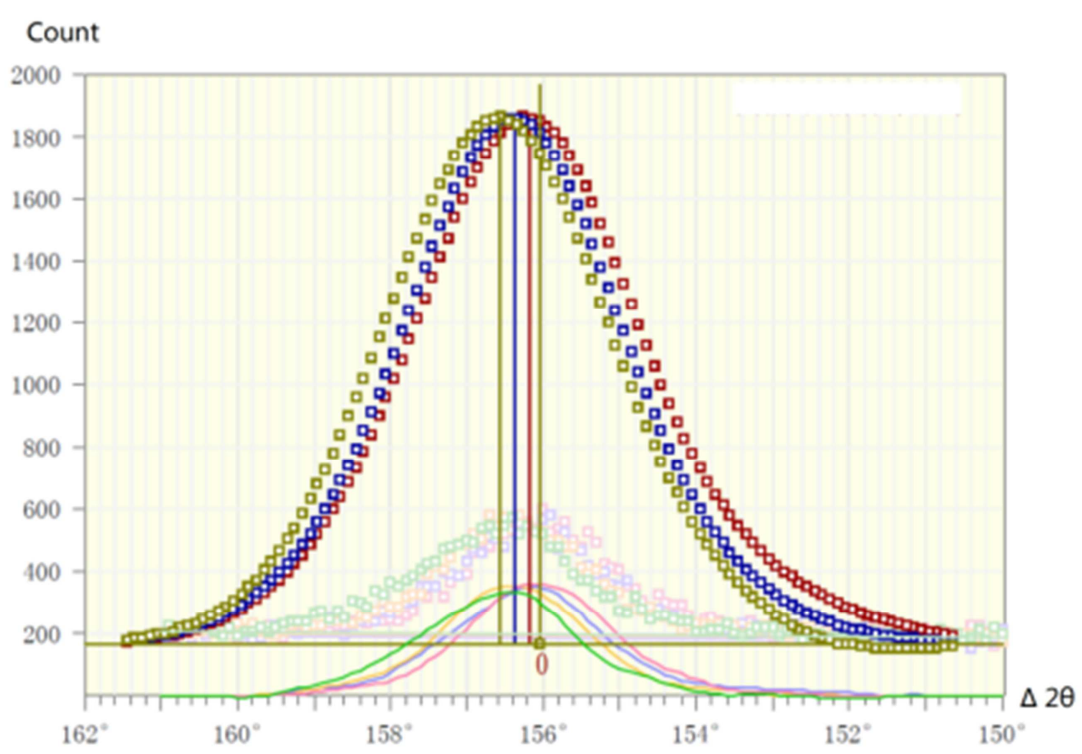

Figure 2. Distribution curves of the correlation function.

The (20) $\Psi-\sin ^{2} \Psi$ curve obtained from the results of cross-correlation function at the point 7 of the specimen in Figure 1 is shown in Figure 3. It can be seen that the (20) $\Psi$ - $\sin ^{2} \Psi$ curve at this point has a good fit and a small error, proving that the testing results are reliable.

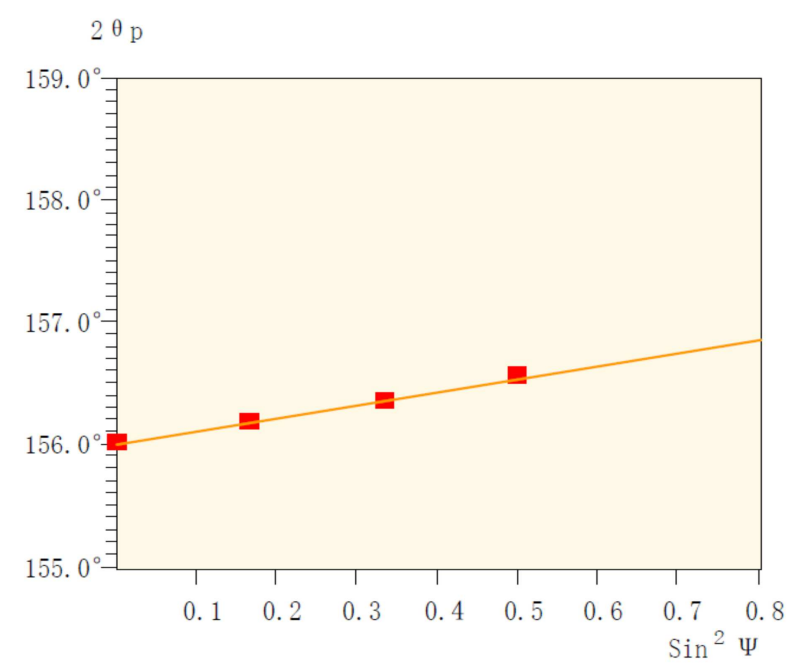

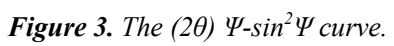

\section{Specimens for Measuring Residual Stress by X-ray Diffraction}

The influence factors for measuring the residual stress of the specimen are mainly manifested in the following three aspects: correct sampling (related to the distribution of testing points), the protection of specimen, and the structure of specimen. There are no strict regulations on the shape, size and quality of the specimen for measuring residual stress by $\mathrm{X}$-ray diffraction. However, the testing points of the specimen should have sufficient space and angle range for the equipment. The state of the specimen will affect the testing results of the residual stress. Therefore, the surface residual stress of the specimen can be accurately obtained by protecting the state of the specimen.

\subsection{Correct Sampling}

According to the testing conditions in Table 1, the residual stress of the specimen shown in Figure 1 is measured. The longitudinal residual stress distribution curve of the specimen is shown as the dotted curve in Figure 4. Then the specimen was cut at the point 10 and the residual stress of the specimen is measured again at the original testing point. The longitudinal residual stress distribution curve of the specimen is shown as the square curve in Figure 4.

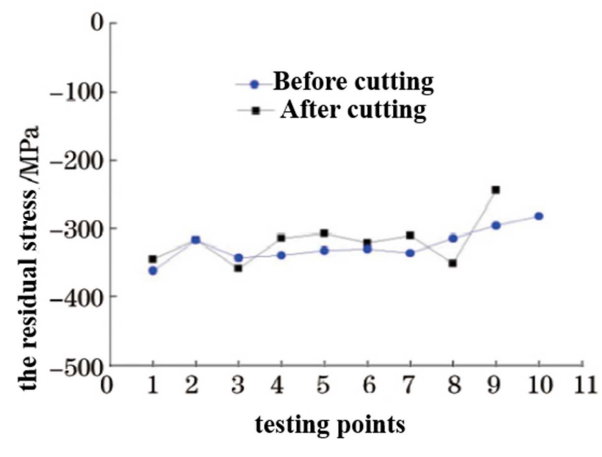

Figure 4. Distribution curves of the residual stress.

Figure 4 shows that the distribution curves of longitudinal residual stress of the specimen at the point 1 to 8 are basically 
the same before and after the cutting. However, the residual stress $(51.9 \mathrm{MPa})$ at the point 9 is lower than that before the cutting due to the stress release after the cutting. Therefore, for specimens with specific requirements for the testing location of the residual stress, the effect of the cutting process on the residual stress release should be considered before cutting. In order to ensure the validity and accuracy of the testing results, a certain cutting allowance (generally more than $10 \mathrm{~mm}$ ) must be left.

\subsection{Protection State of the Specimen}

The distribution curves of the longitudinal residual stress at the point 1 to 8 after hammer peening around at the point 2 and 4 are shown in Figure 5. Figure 5 shows that the longitudinal residual stress increases significantly after the specimen peened by hammer. In particular, the residual stress changes from -316.9 to $-374.7 \mathrm{MPa}$ and the compressive stress has increased by $57.8 \mathrm{MPa}$ at the point 2 . The residual stress changes from-315.0 to-378.7 $\mathrm{MPa}$ and the compressive stress increases by $63.7 \mathrm{MPa}$ at the point 4 .

In the testing process of the residual stress, the surface near the test point may be subjected to external forces such as mechanical damage or deformation. For example, the specimen needs to be clamped on the table, and the process may have an effect on testing results. Therefore, the surface of the specimen should be properly protected to avoid mechanical bruises and scratches during the transportation and additional stress during clamping. At the same time, there should be no dirt, oil film, thick oxide layer, etc. When the testing surface does not meet the above requirements, it must be cleaned and electro-polished.

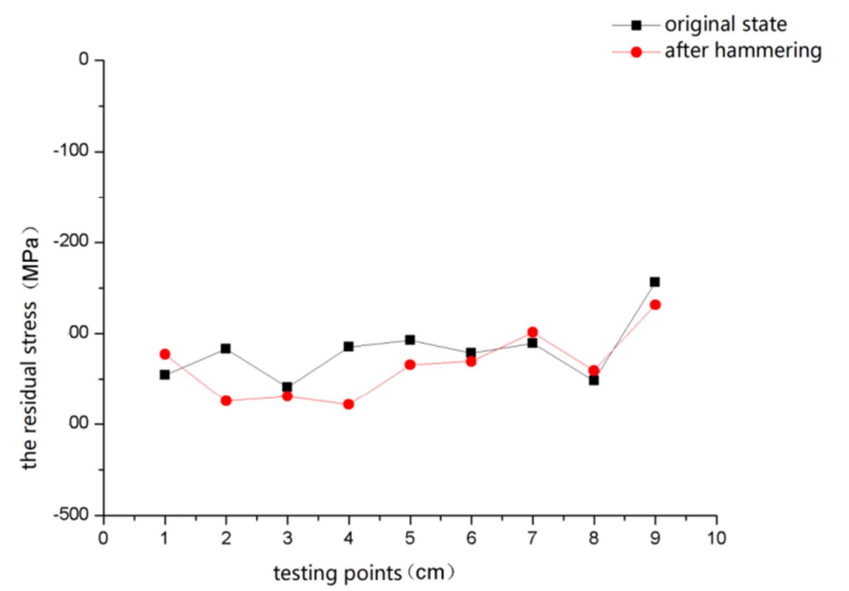

Figure 5. Distribution curves of the residual stress after hammer peening.

\subsection{Structure State of the Specimen}

The conventional X-ray diffraction method for measuring residual stress requires a homogeneous, continuous and isotropic material. SAVALONI et al. [15], found that the $<111>$ texture tends to tensile stress state while the $<200>$ texture tends to compressive stress state. Generally, there is some texture in many thin film coatings, which has an impact on the testing results of the residual stress. The addition of oscillation angle can effectively improve the accuracy of the testing results.

\section{Conclusions and Recommendations}

The X-ray diffraction method, as an effective non-destructive testing method, is used to determine the residual stress accurately, and its theory is quite mature. The main influence factors on the accuracy of the testing results in the testing process are as follows.

1. It is crucial to correctly determine the properties of the material, which ensures that corresponding reasonable testing parameters can be set.

2. The sampling process and specimen protection can also affect the testing results of the residual stress.

3. The structure state of the materials will have a great impact on testing results. For example, the texture will cause excessive errors of the testing results.

In order to ensure the stability and accuracy of the testing results, it is recommended to understand the material properties in detail before testing, to ensure sufficient margin when sampling, to apply the strict protection for the test surface of the sample, and to add a swing angle appropriately when the material with texture.

\section{References}

[1] TAN Sen. Estimating surface residual stress based on indentation and finite element simulation [D]. Harbin: Harbin Institute of Technology, 2006: 33-34.

[2] CUI Keqing. Safety engineering dictionary [M]. Beijing: Chemistry Industry Press, 1995: 125-154.

[3] XU Shunsheng, The study on metal by X-ray [M]. Shanghai: Shanghai Scientific \& Technical Publishers, 1962: 570.

[4] ZHOU Shangqi, The analysis of X-ray diffraction [M]. Chongqing: Chongqing University Press, 1991: 200.

[5] CHENG Xiaoyu, WANG Xiaomei. Essence of residual stress and adjust [J]. Total corrosion control. 2009, 23 (7): 33-35.

[6] JIANG Gang, Present Research Status of Measuring Residual Stress [J] Machine Tool \& Hydraulics, 2007, Vol 6, 213-216.

[7] LIU Jinna, XU Binshi, WANG Haidou. Development tendency of measurement methods for residual stresses [J]. Physical testing and chemical analysis part a: physical testing, 2013, 49 (10): 677-682.

[8] CHEN Yu-an, ZHOU Shangqi, The-state-of-the-art of the study on residual stress measurement by $\mathrm{X}$-ray analysis [J]. Nondestructive testing, 2001, 23 (1): 19-22.

[9] GB/T 7704-2017 Non-destructive testing---practice for residual stress measurement by X-ray [S].

[10] SHI Xinhua, WU Lihong, LI Chun. Introduction to the latest European and American standard test methods for residual stress measurement by X-ray diffraction [J]. Physical testing and chemical analysis part A: physical testing, 2011, 47 (10): 623-628. 
[11] EN 15305: 2008 Non-destructive testing---Test method for residual stress analysis by X-ray diffraction [S].

[12] ASTM E915-2016 Standard test method for verifying the alignment of X-ray diffraction instrumentation for residual stress measurement [S].

[13] ZHANG Dingquan, Basic knowledge of stress determination by X-ray - lecture No. 1 basic concept of residual stress [J]. Physical testing and chemical analysis part a: physical testing, 2007, 43 (4): 211-212.
[14] LV Kemao. Basic knowledge of residual stress determination-Lecture No. 4 stress determination method by $\mathrm{X}$-ray (I) $[\mathrm{J}]$. Physical testing and chemical analysis part a: physical testing, 2007, 43 (7): 349-354.

[15] SAVALONI H, TAHERIZADEH A, ZENDEHNAM A. Residual stress and structural characteristics in $\mathrm{Ti}$ and $\mathrm{Cu}$ sputtered films on glass substrates at different substrate temperatures and film thickness [J]. Physical: B, 2004, 349 (1): 44-55. 